\title{
Circular RNA hsa_circ_0011946 promotes the malignant process of salivary adenoid cystic carcinoma by downregulating miR-1205 expression
}

\author{
HUIPING WEI, JUN LI, CHENG XIE and HAO DONG \\ Department of Oral and Maxillofacial Surgery, The People's Hospital of Guangxi Zhuang Autonomous Region, \\ Nanning, Guangxi Zhuang Autonomous Region 530021, P.R. China
}

Received October 14, 2020; Accepted March 18, 2021

DOI: $10.3892 /$ etm.2022.11224

\begin{abstract}
Circular RNA (circRNA/circ) hsa_circ_0011946 has been reported to serve an important role in a number of cancer types; however, to the best of our knowledge, its role in salivary adenoid cystic carcinoma (SACC) has not been reported. In the present study, the primary focus was the effects of hsa_circ_0011946 on the invasion, migration and epithelial-mesenchymal transformation (EMT) of SACC cells, and the specific mechanisms involved. The expression levels of hsa_circ_0011946 and microRNA (miR)-1205 in cancer tissues and paracancerous tissues of patients with SACC were analyzed using reverse transcription-quantitative (RT-q) PCR. The cell proliferation rate was determined using a Cell Counting Kit-8 assay. Wound healing assays were performed to analyze the cell migratory ability, while a transwell assay was used to measure the cell invasion ability. Western blotting was used to analyze the expression levels of EMT-related proteins. Cell transfection was used to knockdown hsa_circ_0011946 and knockdown or overexpress miR-1205. Subcellular localization assays for hsa_circ_0011946 were performed using RT-qPCR. A dual-luciferase reporter gene assay was used to verify the binding between hsa_circ_0011946 and miR-1205. The results of the present study revealed that the expression levels of hsa_circ_0011946 were significantly upregulated in cancer tissues from patients with SACC. The knockdown of hsa_circ_0011946 expression inhibited the proliferation, invasion and migration of SACC cells, thereby inhibiting the EMT process, which was achieved by downregulating miR-1205 expression. In conclusion, circRNA hsa_circ_0011946 was
\end{abstract}

Correspondence to: Dr Hao Dong, Department of Oral and Maxillofacial Surgery, The People's Hospital of Guangxi Zhuang Autonomous Region, 6 Taoyuan Road, Qingxiu, Nanning, Guangxi Zhuang Autonomous Region 530021, P.R. China

E-mail: donghaoa2020@163.com

Key words: salivary adenoid cystic carcinoma, competing endogenous RNA, hsa_circ_0011946, microRNA-1205, malignant progression of tumor discovered to promote the malignant process of SACC by downregulating miR-1205 expression.

\section{Introduction}

Adenoid cystic carcinoma (ACC), also known as cylindrical tumor or cylindrical adenocarcinoma, is one of the most common types of malignant tumor found in the salivary glands. ACC tumors are round or nodular in shape, with unclear boundaries between the mass and surrounding tissue (1). Salivary ACC (SACC) accounts for 5-10\% of salivary gland tumors, $24 \%$ of salivary gland malignancies and is usually located within the salivary glands (2). Although SACC may occur at any age, it predominately occurs in middle-aged and elderly individuals (aged 40-60 years), with no discrepancies in the incidence rates between males and females and this is more common in 40-60-year olds compared with individuals older than 60. SACC is a slow-growing salivary epithelial tumor but has a high malignant potential. Due to the resistance of SACC to traditional treatment regimens, SACC is characterized by a strong local invasive potential, high rates of hematogenous metastasis and a poor long-term prognosis (3).

A previous study revealed that hsa_circular RNA (circRNA/circ)_0011946 promoted the migration and invasion of hepatocellular carcinoma by inducing epithelial-mesenchymal transition (EMT) (4). hsa_circ_0011946 also promoted the growth, migration and invasion of oral squamous cell carcinoma cells by upregulating proliferating cell nuclear antigen expression (5). The knockdown of hsa_circ_0011946 also inhibited the migration and invasion of MCF-7 breast cancer cells by targeting replication factor $\mathrm{C}$ subunit 3 (6). However, to the best of our knowledge, the role of has_circ_0011946 in SACC has not been reported.

miR-1205 has previously been reported to play an anticancer role in ovarian cancer (7), gastric cancer (8), laryngeal squamous cell carcinoma (9), colorectal cancer (10), thyroid papillary carcinoma (11). However, to the best of our knowledge, the role of miR-1205 in SACC has not been reported. Therefore, the present study aimed to analyze the expression levels of hsa_circ_0011946 and miR-1205 in SACC and to determine the specific underlying mechanisms in order to provide potential novel targets for the treatment of SACC. 


\section{Materials and methods}

Clinical samples. The samples of patients (two male and three female; aged $45 \pm 0.26$ years) with SACC selected in this paper were from The People's Hospital of Guangxi Zhuang Autonomous Region (Nanning, China) from January 2019 to January 2020. There were five cancerous and five paracancerous tissues $5 \mathrm{~cm}$ away from tumor tissue in total from five patients. The experiments were approved by the Ethics Committee of The People's Hospital of Guangxi Zhuang Autonomous Region, and informed consent was signed by all participants. The inclusion criteria used were as follows: i) Patients that met pathological diagnostic criteria of WHO and the diagnosis was clear; and ii) patients who had the first onset. The exclusion criteria used were as follows: i) Patients with malignant tumor of other organs; ii) patients who received prereoperative radiotherapy or chemotherapy; and iii) patients who disagreed to be observed.

Cell lines and culture. The SACC cell line SACC-LM was obtained from The Cell Bank of Type Culture Collection of The Chinese Academy of Sciences. Cells were cultured in DMEM (Gibco; Thermo Fisher Scientific, Inc.) supplemented with 10\% FBS (Gibco; Thermo Fisher Scientific, Inc.), and maintained at $37^{\circ} \mathrm{C}$ with $5 \% \mathrm{CO}_{2}$. After digestion with trypsin at $37^{\circ} \mathrm{C}$ for $2 \mathrm{~min}$ (Gibco; Thermo Fisher Scientific, Inc.), the cells were separated in a low-speed centrifuge at $4^{\circ} \mathrm{C}$ for 10 min (300 x g, Hennuo Instrument Equipment Co., Ltd.) and then the subsequent experiments were performed.

Reverse transcription-quantitative PCR (RT-qPCR). Cells were collected $48 \mathrm{~h}$ after transfection with shRNA-hsa circ_0011946 and by reference to the guidance of the manufacturer (Ambion; Thermo Fisher Scientific, Inc.), cells were segregated by means of a PARIS ${ }^{\mathrm{TM}}$ kit. Concisely, cells underwent lysis in $1 \mathrm{ml}$ cell segregation buffer and $15 \mathrm{~min}$ of centrifugation at $500 \mathrm{xg}$ at $4^{\circ} \mathrm{C}$. RNA was then extracted from the nuclear pellet and cell supernatant using TRIzol LS and and TRIzol ${ }^{\circledR}$ reagent (Invitrogen; Thermo Fisher Scientific, Inc.). RNA concentration and quantification were assessed using a NanoDrop spectrophotometer (Thermo Fisher Scientific, Inc.). Total RNA was reverse transcribed into cDNA using an Invitrogen SuperScript ${ }^{\mathrm{TM}}$ III Reverse Transcriptase kit (Thermo Fisher Scientific, Inc.) according to the manufacturer's protocols. qPCR was subsequently performed using SYBR Green qPCR Master mix (Takara Biotechnology Co., Ltd.) on a StepOnePlus Real-Time PCR System (Thermo Fisher Scientific, Inc.). hsa_circ_0011946 and miR-1205 expression levels were analyzed using qPCR and normalized to U6 small nuclear RNA expression as the internal control. The following thermocycling conditions were used for qPCR: $95^{\circ} \mathrm{C}$ for $10 \mathrm{~min}$; followed by 40 cycles of $95^{\circ} \mathrm{C}$ for $10 \mathrm{sec}$ and $60^{\circ} \mathrm{C}$ for $60 \mathrm{sec}$. The following primer sequences were used for the qPCR: hsa_circ_0011946 forward, 5'-GCTGGTGTTCCT TGACTGGA-3' and reverse, 5'-CACTGTAGCAAACCAGCA TTTCT-3'; miR-1205 forward, 5'-CACGCATCTGCAGGG TTT-3' and reverse, 5'-CCAGTGCAGGGTCCGAGGTA-3'; and U6 forward, 5'-GCGCGTCGTGAAGCGTTC-3' and reverse, 5'-GTGCAGGGTCCGAGGT-3'. hsa_circ_0011946 and miR-1205 expression levels were normalized to U6 small nuclear RNA. Fold-changes in circRNA expression and miR-1205 were calculated using the $2^{-\Delta \Delta C q}$ method (12).

Cell transfection. miR-1205 mimics and negative control (NC) mimic, miR-1205 inhibitor and NC inhibitor, short hairpin RNA (shRNA)-hsa_circ_0011946-1, shRNA-hsa_circ_0011946-2 and shRNA-NC were purchased from Shanghai GenePharma Co., Ltd. The expression of hsa_circ_0011946-1 gene was knocked down by shRNA using lentiviral expression vector GV 493 (Shanghai GenePharma Co., Ltd.). Cells were transfected with shRNA-hsa_circ_0011946, miR-1205 mimics and NC mimic, miR-1205 inhibitors and NC inhibitor all at a concentration of $20 \mathrm{nM}$ using Lipofectamine ${ }^{\circledR} 2000$ reagent (Invitrogen; Thermo Fisher Scientific, Inc.) at $37^{\circ} \mathrm{C}$ for $48 \mathrm{~h}$ according to the manufacturer's protocol. The sequences used were miR-1205 mimic (5'-UCUGCAGGGUUUGCUUUGAG-3') and NC mimic (5'-UGACGUCGGUUUGCUUUGAG-3'); miR-1205 inhibitor (5'-CUCAAAGCAAACCCUGCAGA-3') and NC inhibitor (5'-CAGUACUUUUGUGUAGUACAAA-3'). RT-qPCR was used to detect the transfection efficiency $48 \mathrm{~h}$ after transfection.

Cell Counting Kit-8 (CCK-8) assay. Transfected cells $\left(4 \times 10^{3}\right.$ cells/well) were seeded into 96 -well plates for 24,48 or $72 \mathrm{~h}$. Following the incubation, cells were incubated with $10 \mu \mathrm{l} \mathrm{CCK}-8$ for a further $4 \mathrm{~h}$ at $37^{\circ} \mathrm{C}$. The absorbance at a wavelength of $450 \mathrm{~nm}$ was measured using a microplate reader (VersaMax; Beijing Yuechangxing Technology Co., Ltd.).

Wound healing assay. Cells were seeded into six-well plates and cultured in DMEM with 10\% FBS to 80\% confluence, before the cell monolayers were scratched with sterile plastic pipette tips (width, $2 \mathrm{~mm}$ ) and washed with PBS to remove cell debris. Cells were incubated in serum-free DMEM at $37^{\circ} \mathrm{C}$ and then imaged at 0 and $24 \mathrm{~h}$ using a light microscope (magnification, $\mathrm{x} 100)$ in five randomly selected fields of view. The cell migratory distance into the wound area was calculated using ImageJ 1.48 software (National Institutes of Health). The percentage of wound closure was determined according to the following equation: [(Ai-At)/Ai] x100, where Ai represents the initial area of the wound at $0 \mathrm{~h}$ and At represents the area of the wound after $24 \mathrm{~h}$.

Transwell assay. Cell suspensions were plated into the upper chambers of 24 -well Transwell chambers $(8-\mu \mathrm{m}$ pore size; Corning, Inc.) at a density of $1-1.5 \times 10^{6}$ cells $/ \mathrm{ml}$. The lower chambers were filled with $0.5 \mathrm{ml}$ DMEM supplemented with $10 \%$ FBS. The upper chambers were precoated with Matrigel (1:8, BD Biosciences) for the invasion assay at $37^{\circ} \mathrm{C}$ for $24 \mathrm{~h}$, according to the manufacturer's protocol. Following incubation for $24 \mathrm{~h}$ at $37^{\circ} \mathrm{C}$, cells remaining in the upper chamber of the membrane were removed using cotton swabs cells, while cells in the lower chamber were fixed with $10 \%$ paraformaldehyde at room temperature for $15 \mathrm{~min}$ and stained with $0.1 \%$ crystal violet for $30 \mathrm{~min}$ at room temperature. Stained cells were counted under a light microscope (magnification, x100). Cells on the bottom surface were quantified by counting five random fields per well using ImageJ software version 146 (National Institutes of Health), and the mean number of cells passing through the chamber were calculated. Three independent experiments were performed, and the data are presented as the average \pm SD. 
A

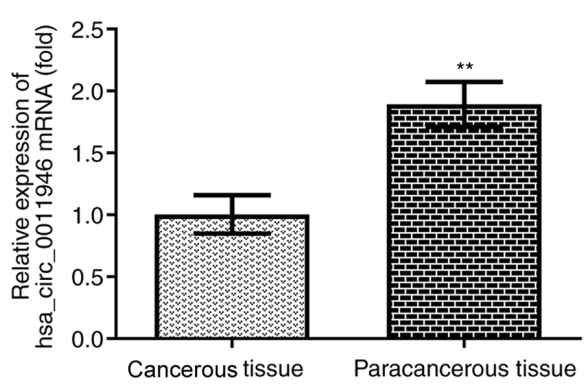

B

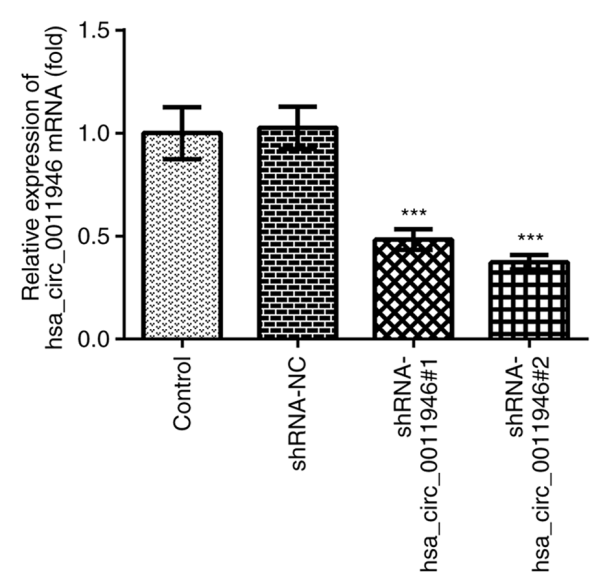

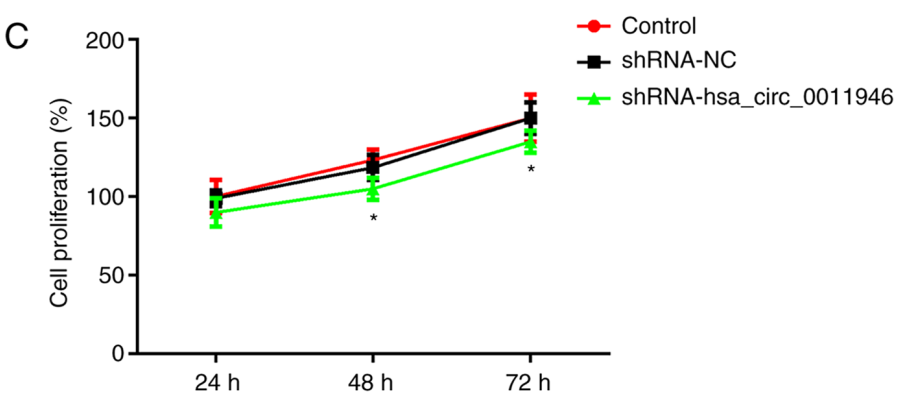

Figure 1. Knockdown of Hsa_circ_0011946 inhibits proliferation of SACC cells. (A) RT-qPCR detected the expression of Hsa_circ_0011946 in cancerous and paracancerous tissues from patients with SACC. ${ }^{* *} \mathrm{P}<0.01$ vs. cancerous tissues. (B) RT-qPCR was performed to detect the expression of Hsa_circ_0011946 in SACC cells after the transfection in the SACC-LM cells. ${ }^{* * *} \mathrm{P}<0.001$ vs. shRNA-NC. (C) Cell Counting Kit- 8 detected the cell viability in the SACC-LM cells. $\mathrm{P}<0.05$ vs. shRNA-NC. Circ, circular; RT-qPCR, reverse transcription-quantitative PCR; NC, negative control; SACC, salivary adenoid cystic carcinoma; sh, short hairpin.

Western blotting. Total protein was extracted from each group of cells using RIPA lysis buffer (Sigma-Aldrich; Merck KGaA) at $48 \mathrm{~h}$ after cell transfection. Total protein was quantified using a bicinchoninic acid (BCA) protein assay kit (Bio-Rad Laboratories, Inc.). Protein (30 $\mu \mathrm{g} /$ lane) was separated via $10 \%$ SDS-PAGE, then transferred onto polyvinylidene difluoride membranes (Bio-Rad Laboratories, Inc.) that were blocked with $5 \%$ non-fat milk for $1 \mathrm{~h}$ at room temperature. The membranes were subsequently incubated with the following primary antibodies overnight at $4^{\circ} \mathrm{C}$ : Anti-E-cadherin $(1: 1,000$; cat. no. ab40772; Abcam), anti-N-cadherin (1:1,000; cat. no. ab76011; Abcam), anti-vimentin (1:1,000; cat. no. ab92547; Abcam) and anti-GAPDH (1:1,000; cat. no. ab205718; Abcam). Following the primary antibody incubation, the membranes were washed with PBS and incubated with HRP-conjugated anti-rabbit secondary (1:5,000; cat. no. ab205718; Abcam) for $1 \mathrm{~h}$ at room temperature. Protein bands were visualized using an enhanced chemiluminescence detection system (EMD Millipore). Densitometric analysis was performed using ImageJ software version 146 (National Institutes of Health). The ratio of the gray value of the target protein band to that of GAPDH was regarded as the relative protein expression.

Target prediction. circRNA interactome (https://circinteractome.irp.nia.nih.gov) was used to predict the binding sites between hsa_circ_0011946 and miR-1205.

Dual luciferase reporter assay. miR-1205 mimic or NC mimic were co-transfected with wild-type or mutant
hsa_circ_0011946-Luc plasmids all at a concentration of $20 \mathrm{nM}$ into SACC-LM cells for $48 \mathrm{~h}$ using Lipofectamine $2000^{\circledR}$ (Invitrogen; Thermo Fisher Scientific, Inc.). The cells were subsequently washed with PBS and lysed using Passive Lysis Buffer (Promega Corporation). After transfection for $48 \mathrm{~h}$, the relative luciferase activity was measured using a microplate reader (BD Biosciences) and normalized to Renilla luciferase activity, which was measured using a Renilla luciferase activity kit (pRL-TK; Invitrogen; Thermo Fisher Scientific Inc.). The procedure was performed according to the manufacturer's protocols. All plasmids were constructed by Thermo Fisher Scientific, Inc.

Statistical analysis. All data are presented as the mean \pm SD. All experiments were repeated three times. A one-way ANOVA followed by Tukey's post hoc test was used to determine the statistical differences between multiple groups. $\mathrm{P}<0.05$ was considered to indicate a statistically significant difference.

\section{Results}

hsa_circ_0011946 expression levels are upregulated in cancer tissues of patients with SACC. RT-qPCR was used to analyze the expression levels of hsa_circ_0011946 in cancerous tissues of patients with SACC. The results revealed that, compared with paracancerous tissue, the expression levels of hsa_circ_0011944 were significantly upregulated in SACC tissue (Fig. 1A). Subsequently, SACC-LM cells were selected for use in subsequent experiments. 
A
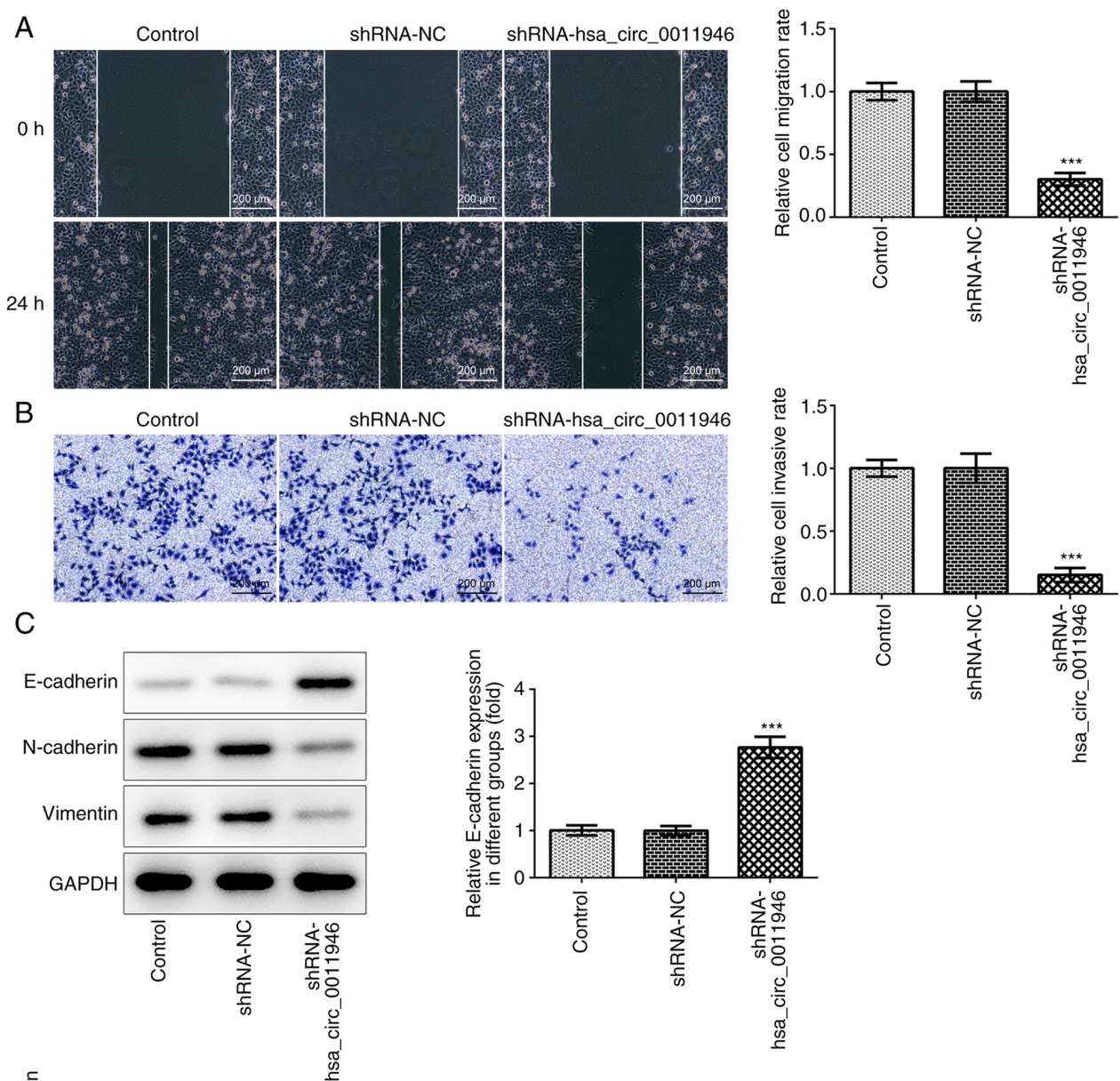

ShRNA-NC

shRNA-hsa_circ_0011946
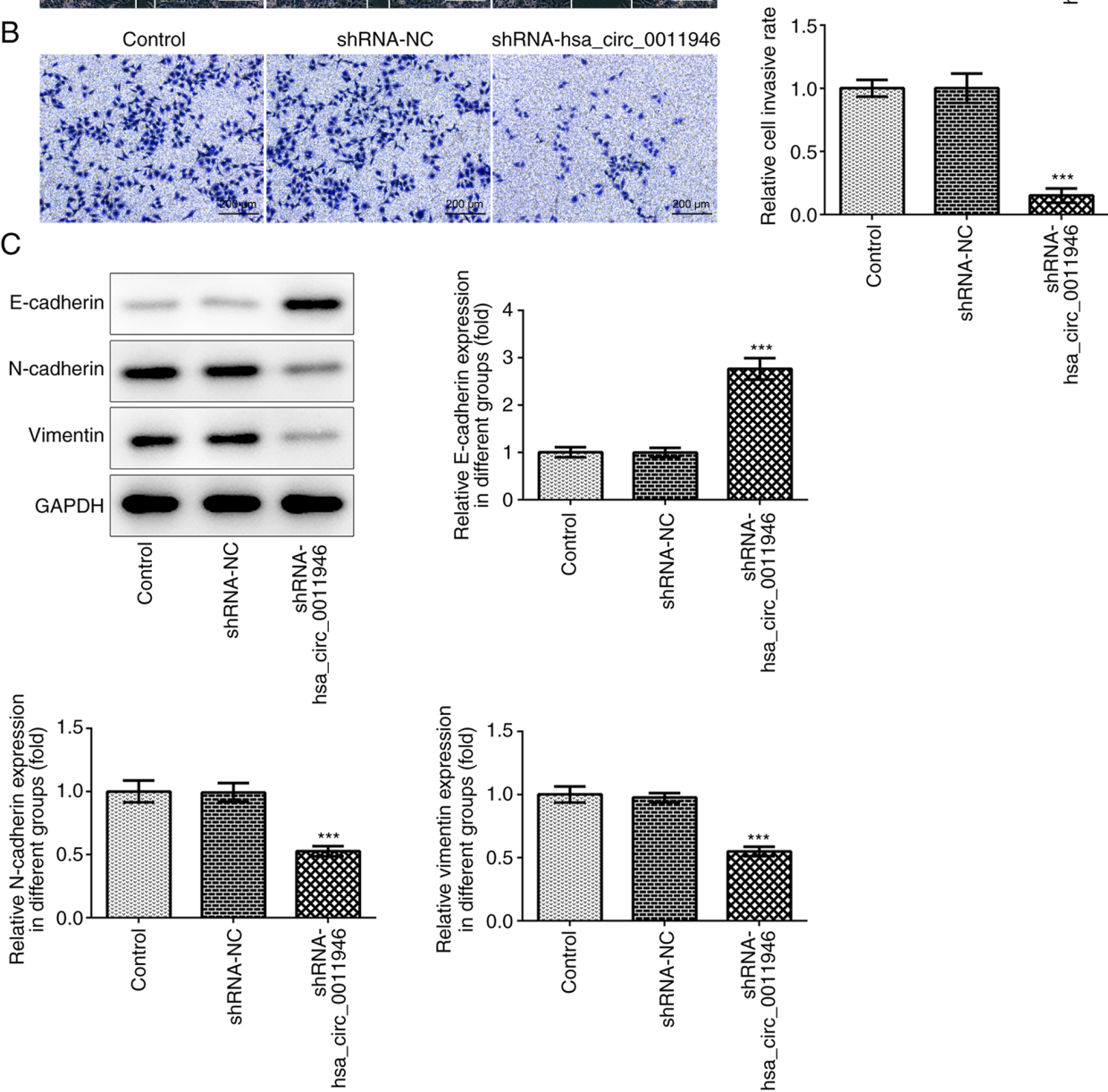

Figure 2. Knockdown of Hsa_circ_0011946 inhibits invasion, migration and EMT processes in SACC cells. (A) A wound healing assay was performed to detect the cell migration ability (magnification, $\mathrm{x} 100$ ). (B) Transwell assays were performed to detect the cell invasion ability (magnification, $\mathrm{x} 100$ ). (C) Western blot analysis was performed to detect the expression of EMT-related proteins. ${ }^{* * *} \mathrm{P}<0.001 \mathrm{vs}$. shRNA-NC. EMT, epithelial-mesenchymal transition; Circ, circular; NC, negative control; SACC, salivary adenoid cystic carcinoma; sh, short hairpin.

Knockdown of hsa_circ_0011946 inhibits the proliferation of SACC cells. Cell transfection experiments were used to interfere with the expression levels of hsa_circ_0011946 in SACC-LM cells, and RT-qPCR was used to verify the transfection efficiency (Fig. 1B). The results demonstrated that the expression levels of hsa_circ_0011946 were significantly downregulated in shRNA-hsa_circ_0011946-1- and shRNA-hsa_circ_0011946-2-transfected cells compared with the shRNA-NC group, and this was more notable in the shRNA-hsa_circ_0011946-2 group. Therefore, shRNA-hsa_ circ_0011946-2 was selected for use in subsequent experiments. The cells were then divided into the following three groups: i) Control group; ii) shRNA-NC group; and iii) shRNA-hsa circ_0011946 group. CCK-8 assays were used to determine the cell proliferation rate. Compared with the shRNA-NC group, no statistically significant differences were observed in the cell proliferation rate following $24 \mathrm{~h}$ of transfection with shRNA-hsa_circ_0011946. However, following 48 and $72 \mathrm{~h}$ of interference, the cell proliferation ability was significantly decreased compared with the shRNA-NC group (Fig. 1C). These results suggested that the knockdown of hsa_circ_0011946 expression may inhibit the proliferation of SACC cells.

Knockdown of hsa_circ_0011946 inhibits invasion, migration and EMT processes in SACC cells. The invasive and migratory abilities of cells following the knockdown of hsa_circ_0011946 were analyzed, and the results revealed that the invasive (Fig. 2A) and migratory (Fig. 2B) abilities 


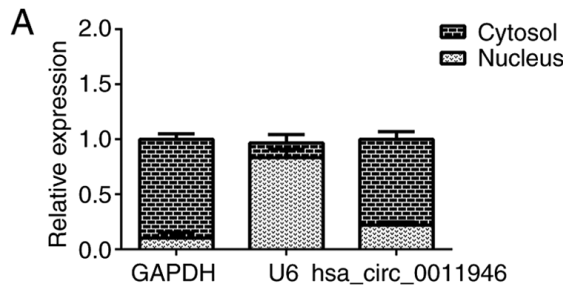

\author{
B \\ hsa_circ_0011946 5' CUUGACUGGAGACAACCUGCAGC...3 $3^{\prime}$ \\ | I I I I I I \\ hsa-ImiR-1205 3' GAGUUUCGUUUGGGACGUCU...5 $5^{\prime}$
}
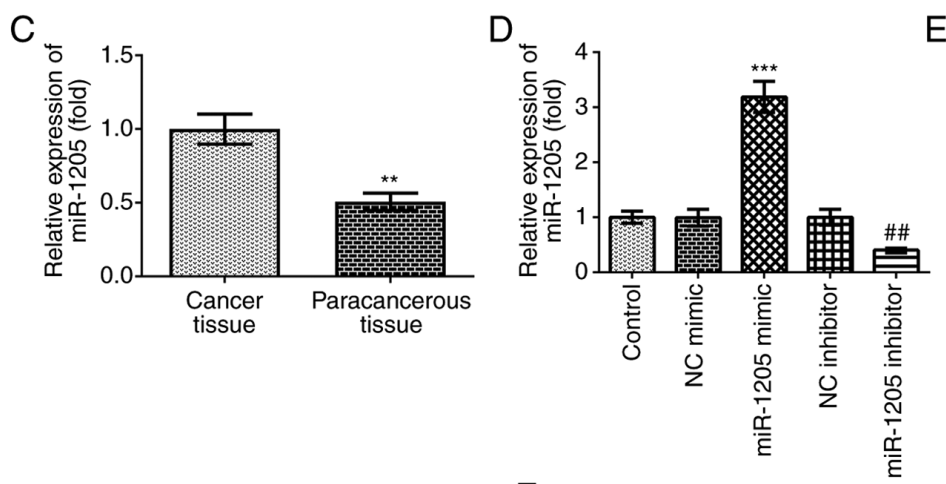

E

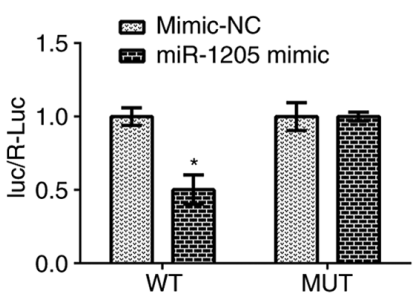

$\mathrm{F}$

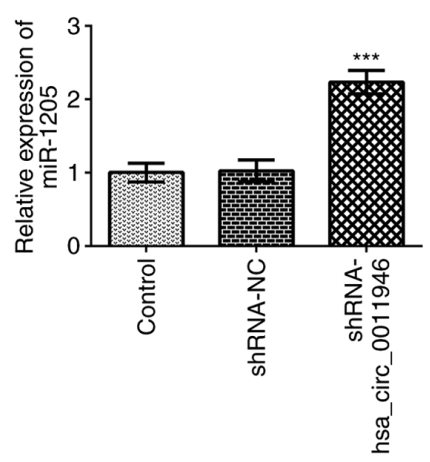

Figure 3. Hsa_circ_0011946 regulates the expression of miR-1205 negatively via sponge-like adsorption. (A) The expression of Hsa_circ_0011946 is detected through RT-qPCR. (B) Targeted binding sites of Hsa_circ_0011946 and miR-1205. (C) RT-qPCR detected the expression of miR-1209 in cancer tissues and paracancerous tissues of patients with SACC. ${ }^{* *} \mathrm{P}<0.01$ vs. cancer tissues. (D) RT-qPCR detected the expression of miR-1205 in SACC cells after the transfection. ${ }^{* * * *} \mathrm{P}<0.001$ vs. NC mimic, ${ }^{\# \#} \mathrm{P}<0.001$ vs. NC inhibitor. (E) The luciferase reporter gene verified the targeted binding of Hsa_circ_0011946 to miR-1205. ${ }^{*} \mathrm{P}<0.05$ vs. mimic-NC. (F) RT-qPCR detected the expression of miR-1209 after interfering with Hsa_circ_0011946 expression. ${ }^{* * *} \mathrm{P}<0.001$ vs. shRNA-NC. Circ, circular; RT-qPCR, reverse transcription-quantitative PCR; NC, negative control; SACC, salivary adenoid cystic carcinoma; WT, wild type; Mut, mutant.

of cells in the shRNA-hsa_circ_0011946 group were significantly decreased compared with the shRNA-NC group. The expression levels of the following EMT-related proteins were analyzed using western blotting: E-cadherin, $\mathrm{N}$-cadherin and vimentin (Fig. 2C). Compared with the shRNA-NC group, the expression levels of E-cadherin were significantly upregulated in the shRNA-hsa_circ_0011946 group, while the expression levels of $\mathrm{N}$-cadherin and vimentin were significantly downregulated, suggesting that hsa_circ_0011946 interfered with the EMT process in SACC cells.

hsa_circ_0011946 negatively regulates the expression levels of miR-1205 by acting as a sponge. The presence of hsa_circ_0011946 was confirmed by RT-qPCR (Fig. 3A). The binding site between hsa_circ_0011946 and miR-1205 was predicted using circRNA interactome tools (Fig. 3B). The expression levels of miR-1205 were subsequently found to be significantly downregulated in SACC tissues compared with paracancerous tissue (Fig. 3C). RT-qPCR analysis revealed that cell transfection was successful (Fig. 3D). A dual-luciferase reporter assay was performed to validate the complementary binding between hsa_circ_0011946 and miR-1205 (Fig. 3E). The expression levels of miR-1205 were significantly upregulated following the knockdown of hsa_circ_0011946 (Fig. 3F). These results suggested that hsa_circ_0011946 may negatively regulate the expression levels of miR-1205 by acting as a sponge.

hsa_circ_0011946 promotes the malignant process of SACC by downregulating miR-1205 expression. The cells were divided into control, shRNA-NC, shRNA-hsa circ_001194, shRNA-hsa_circ_0011946 + NC inhibitor and shRNA-hsa_circ_0011946 + miR-1205 inhibitor groups. Cell viability was detected using a CCK- 8 assay and the results revealed that the knockdown of miR-1205 could reverse the inhibition of cell proliferation following the knockdown of hsa_circ_0011946 (Fig. 4A). Compared with shRNA-hsa circ_0011946 + NC inhibitor group, the cell invasive (Fig. 4B) and migratory (Fig. 4C) abilities were significantly increased in the shRNA-hsa_circ_0011946 + miR-1205 inhibitor 

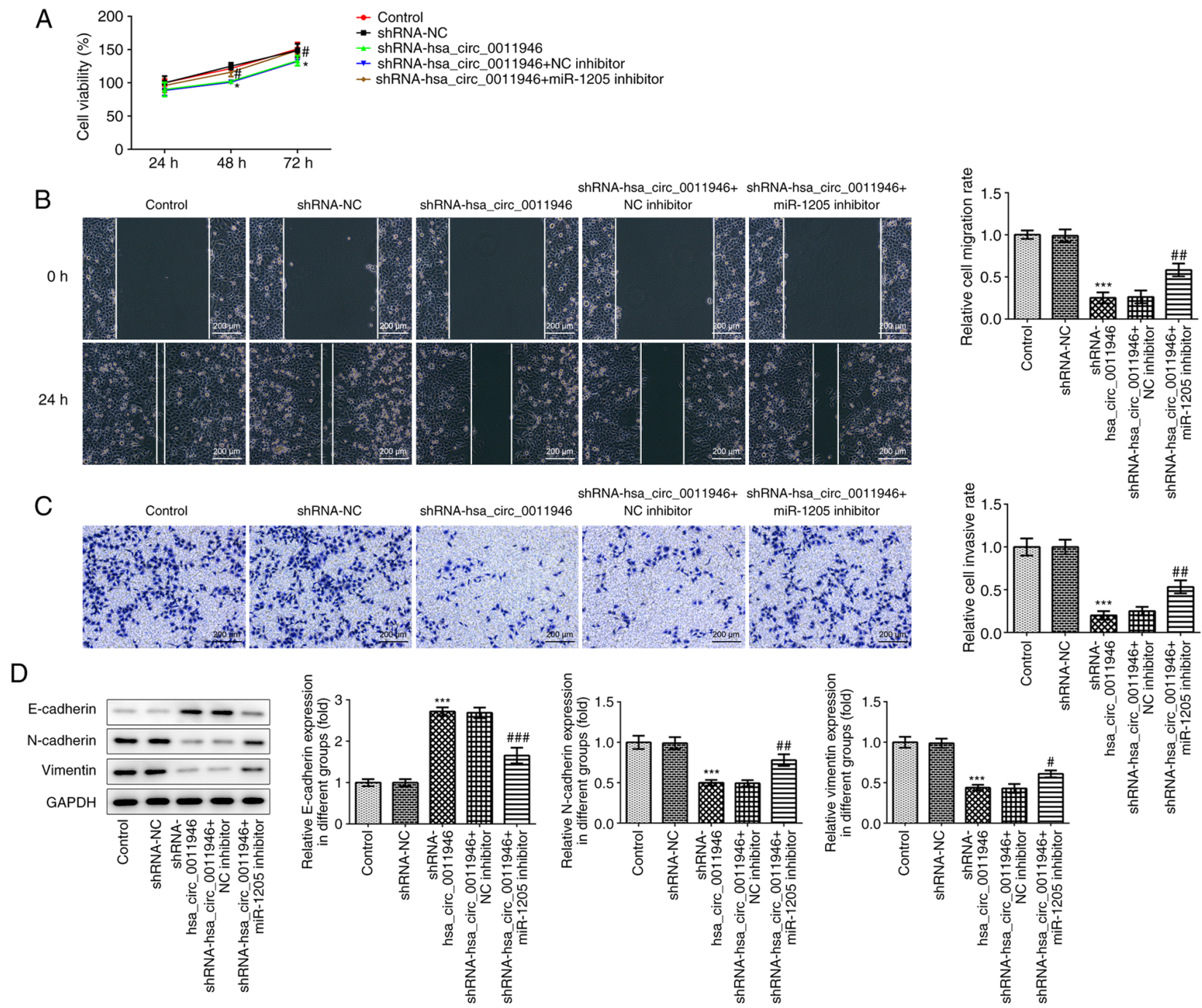

Figure 4. Hsa_circ_0011946 promotes the malignant process of SACC by downregulating miR-1205. (A) Cell Counting Kit-8 detected the cell viability. (B) Wound healing detected the cell migration ability (magnification, $x 100$ ). (C) Transwell detected the cell invasion ability (magnification, x100). (D) Western blot detected the expression of epithelial-mesenchymal transition-related proteins. ${ }^{*} \mathrm{P}<0.05,{ }^{* * *} \mathrm{P}<0.001$ vs. shRNA-NC. ${ }^{\#} \mathrm{P}<0.05,{ }^{\# \#} \mathrm{P}<0.001,{ }^{\# \# "} \mathrm{P}<0.001$ vs. shRNA-hsa_circ_0011946 + NC inhibitor. Circ, circular; NC, negative control; SACC, salivary adenoid cystic carcinoma; miR, micoRNA; sh, short hairpin.

group. Subsequently, the expression levels of EMT-related proteins were analyzed using western blotting. As shown in Fig. 4D, compared with the shRNA-NC group, the expression levels of E-cadherin were significantly upregulated in the shRNA-hsa_circ_0011946 group, while the expression levels of $\mathrm{N}$-cadherin and vimentin were significantly downregulated. The expression levels of E-cadherin protein were downregulated in the shRNA-hsa_circ_0011946 + miR-1205 inhibitor group, while the expression levels of $\mathrm{N}$-cadherin and vimentin were upregulated in the shRNA-hsa circ_0011946 + miR-1205 inhibitor group compared with the shRNA-hsa_circ_0011946 + NC inhibitor group. Altogether, these findings suggested that circRNA hsa_circ_0011946 may promote the malignant progression of SACC by downregulating miR-1205 expression.

\section{Discussion}

SACC is a salivary gland malignancy with high incidence rates and complex occurrence and progression mechanisms, which remain poorly characterized. Following treatment with surgical resection combined with chemoradiotherapy in the clinic, the survival rate of patients with SACC can be improved (13); however, due to the strong invasive nature of the tumor and the neurotropic growth characteristics, recurrence and metastasis often occur following treatment, which seriously affects the quality of life and survival time of patients (14). Therefore, identifying the underlying mechanisms of SACC progression and determining genes or biomarkers associated with the invasion and metastasis of SACC may be of vital importance for the early diagnosis and treatment of patients, in addition to improving prognosis.

circRNAs, members of the non-coding RNA family, are named due to their unique covalently closed circular structure (15). circRNAs are characterized by a strong stability, high abundance and species conservation, as well as demonstrating cell and tissue specificity (16). circRNAs widely exist in the human body and serve an important role in physiological and pathological processes. Currently, circRNAs have been found to be differentially expressed in a variety of cancer types, which 
suggests their potential for use in tumor diagnosis and their potential ability to predict the prognosis of patients (17). Thus, circRNAs are predicted to become novel tumor biomarkers or prognostic indicators. circRNAs regulate gene transcription and translation through various different mechanisms, thereby promoting or inhibiting the proliferation, apoptosis, invasion and metastasis of cancer cells, and ultimately regulating tumor progression (18). The results of the present study revealed that the expression levels of hsa_circ_0011946 were upregulated in cancer tissues of SACC patients. In the present study, no inhibitor of has_circ_0011946 was identified during the literature review, so cell transfection techniques were used to inhibit the expression of has_circ_0011946 in SACC-LM cells $(5,6)$. Following the knockdown of hsa_circ_0011946, the proliferative, invasive and migratory abilities of SACC cells were significantly decreased, and the EMT process was inhibited. The present results also demonstrated that, following the knockdown of hsa_circ_0011946, the proliferation of SACC cells was not significantly inhibited, and the inhibitory effect was only apparent following an extended period of knockdown (48 and $72 \mathrm{~h}$ ). The possible reasons for these results are that SACC cells are locally aggressive (19), thus the knockdown of hsa_circ_0011946 expression may exert little effect on proliferation.

circRNAs have been found to serve a regulatory role through a variety of mechanisms; for example, circRNAs can act as sponges to absorb miRNAs by binding to their miRNA binding sites, and the latter can influence the metabolic process of cells by regulating the expression and activity of target proteins (20). In the present experiments, the knockdown of hsa_circ_0011946 expression targeted and downregulated miR-1205 expression to regulate the malignant progression of SACC cells.

Notably, there were numerous limitations in the current study. Hsa_circ_0011946 was not overexpressed in normal human salivary gland cells, which may have represented an improved study design. In future experiments, the functional differences of the circRNA hsa_circ_0011946 should be investigated in normal HSG and SACC tumor cells.

In conclusion, the results of the present study suggested that the circRNA hsa_circ_0011946 may promote the malignant process of SACC by downregulating miR-1205 expression, which may provide a novel target for the treatment with SACC.

\section{Acknowledgements}

Not applicable.

\section{Funding}

No funding was received.

\section{Availability of data and materials}

The datasets used and/ot analyzed during the current study are available from the corresponding author on reasonable request.

\section{Authors' contributions}

HW and HD wrote the manuscript and analyzed the data. JL and CX performed the experiments and supervised the study.
HD searched the literature and revised the manuscript for important intellectual content. HD and HW confirmed the authenticity of all the raw data. All authors read and approved the final manuscript.

\section{Ethics approval and consent to participate}

The experiments were approved by The Ethics Committee of The People's Hospital of Guangxi Zhuang Autonomous Region, and informed consent was signed by all participants.

\section{Patients consent for publication}

Informed consent was signed by all participants.

\section{Competing interests}

The authors declare that they have no competing interests.

\section{References}

1. Bradley PJ: Adenoid cystic carcinoma evaluation and management: Progress with optimism! Curr Opin Otolaryngol Head Neck Surg 25: 147-53, 2017.

2. Miller JA, An D, Shafique K, Song S, Rao RA, Viswanathan K, Eykman E, Wiles A, Ali SZ, Field A, et al: Mucoepidermoid carcinoma, acinic cell carcinoma, and adenoid cystic carcinoma on fine-needle aspiration biopsy and The Milan System: An international multi-institutional study. J Am Soc Cytopathol 8: 270-277, 2019.

3. Šteiner P, Pavelka J, Vaneček T, Miesbauerová M and Skálová A: Molecular methods for detection of prognostic and predictive markers in diagnosis of adenoid cystic carcinoma of the salivary gland origin. Cesk Patol 54: 132-136, 2018.

4. Ren L, Zhai H, Wang XL, Li JZ and Xia YH: Hsa_circ_0011946 promotes the migration and invasion of hepatocellular carcinoma by inducing EMT process. Eur Rev Med Pharmacol Sci 24: $1108-1115,2020$.

5. Meng Y, Zhao EY, Zhou Y, Qiang DX, Wang S, Shi L, Jiang LY and Bi LJ: Circular RNA hsa_circ_0011946 promotes cell growth, migration, and invasion of oral squamous cell carcinoma by upregulating PCNA. Eur Rev Med Pharmacol Sci 24: 7560, 2020.

6. Zhou J, Zhang WW, Peng F, Sun JY, He ZY and Wu SG: Downregulation of hsa_circ_0011946 suppresses the migration and invasion of the breast cancer cell line MCF-7 by targeting RFC3. Cancer Manag Res 10: 535-544, 2018.

7. Wang G,Zhang HandLiP: Upregulation of hsa_circRNA_102958 indicates poor prognosis and promotes ovarian cancer progression through miR-1205/SH2D3A axis. Cancer Manag Res 12: 4045-4053, 2020.

8. Lin J, Liao S, Li E, Liu Z, Zheng R, Wu X and Zeng W: circCYFIP2 acts as a sponge of miR-1205 and affects the expression of its target gene E2F1 to regulate gastric cancer metastasis. Mol Ther Nucleic Acids 21: 121-132, 2020.

9. Li P, Lin XJ, Yang Y, Yang AK, Di JM, Jiang QW, Huang JR, Yuan ML, Xing ZH, Wei MN, et al: Reciprocal regulation of miR-1205 and E2F1 modulates progression of laryngeal squamous cell carcinoma. Cell Death Dis 10: 916, 2019.

10. Jiang Y, Liu G, Ye W, Xie J, Shao C, Wang X and Li X: ZEB2-AS1 accelerates epithelial/mesenchymal transition through miR-1205/CRKL pathway in colorectal cancer. Cancer Biother Radiopharm 35: 153-162, 2020.

11. Yang Y, Ding L, Li Y and Xuan C: Hsa_circ_0039411 promotes tumorigenesis and progression of papillary thyroid cancer by miR-1179/ABCA9 and miR-1205/MTA1 signaling pathways. J Cell Physiol 235: 1321-1329, 2020.

12. Livak KJ and Schmittgen TD: Analysis of relative gene expression data using real-time quantitative PCR and the 2(-Delta Delta C(T)) method. Methods 25: 402-408, 2001.

13. Laurie SA, Ho AL, Fury MG, Sherman E and Pfister DG: Systemic therapy in the management of metastatic or locally recurrent adenoid cystic carcinoma of the salivary glands: A systematic review. Lancet Oncol 12: 815-824, 2011. 
14. Garg M, Tudor-Green B and Bisase B: Current thinking in the management of adenoid cystic carcinoma of the head and neck. Br J Oral Maxillofac Surg 57: 716-721, 2019.

15. Hsiao KY, Sun HS and Tsai SJ: Circular RNA-new member of noncoding RNA with novel functions. Exp Biol Med (Maywood) 242: 1136-1341, 2017.

16. Han B, Chao J and Yao H: Circular RNA and its mechanisms in disease: From the bench to the clinic. Pharmacol Ther 187: 31-44, 2018.

17. Kristensen LS, Andersen MS, Stagsted LVW, Ebbesen KK, Hansen TB and Kjems J: The biogenesis, biology and characterization of circular RNAs. Nat Rev Genet 20: 675-691, 2019.
18. Vo JN, Cieslik M, Zhang Y, Shukla S, Xiao L, Zhang Y, Wu YM, Dhanasekaran SM, Engelke CG, Cao X, et al: The landscape of circular RNA in cancer. Cell 176: 869-681.e13, 2019.

19. Bouaichi A, Aimad-Eddine S, Mommers XA, Ella B and Zwetyenga N: Intra-mandibular adenoid cystic carcinoma. Rev Stomatol Chir Maxillofac Chir Orale 115: 100-104, 2014.

20. Panda AC: Circular RNAs Act as miRNA sponges. Adv Exp Med Biol 1087: 67-79, 2018

(c) (i) () $९$ This work is licensed under a Creative Commons Attribution-NonCommercial-NoDerivatives 4.0 International (CC BY-NC-ND 4.0) License. 